\title{
Reduced Order vs. Discretized Lumped System Models with Absolute and Relative States for Continuum Manipulators
}

\author{
S.M.Hadi Sadati*, Ali Shiva ${ }^{\dagger \ddagger}$, Ludovic Renson*, Caleb Rucker ${ }^{\S}$, Kaspar Althoefer ${ }^{\Uparrow}$, Thrisantha Nanayakkara ${ }^{\ddagger}$, \\ Christos Bergeles $\|$, Helmut Hauser* and Ian D. Walker** \\ *The Department of Engineering Mathematics, University of Bristol, U.K. \\ Email: s.m.hadi.sadati@bristol.ac.uk \\ ${ }^{\dagger}$ The Department of Informatics, King's College London, U.K. \\ $\ddagger$ The Dyson School of Design Engineering, Imperial College London, U.K. \\ $\S$ The University of Tennessee, Knoxville, TN, USA \\ I The Department of Engineering and Materials Science, Queen Mary University of London, U.K. \\ $\|$ The Department of Imaging and Biomedical Engineering, King's College London, U.K. \\ ${ }^{* *}$ The Department of Electrical and Computer Engineering, Clemson University, SC, USA.
}

\begin{abstract}
A reliable, accurate, and yet simple dynamic model is important to analyze, design and control continuum manipulators. Such models should be fast, as simple as possible and user-friendly to be widely accepted by the ever-growing robotics research community. In this study, we introduce two new modeling methods for continuum manipulators: a general reducedorder model (ROM) and a discretized model with absolute states and Euler-Bernoulli beam segments (EBA). Additionally, a new formulation is presented for a recently introduced discretized model based on Euler-Bernoulli beam segments and relative states (EBR). The models are validated in comparison to experimental results for dynamics of a STIFF-FLOP continuum appendage. Our comparison shows higher simulation accuracy (8-14\% normalized error) and numerical robustness of the ROM model for a system with small number of states, and computational efficiency of the EBA model with near real-time performances that makes it suitable for large systems. The challenges with designing control and observation scenarios are briefly discussed in the end.
\end{abstract}

\section{INTRODUCTION}

Mimicking highly dexterous and deformable biological bodies has been a trending topic of multi-disciplinary research, called soft robotics, using intrinsically soft materials in the form of continuum robotic platforms [12]. However, compliance has disadvantages, such as uncertain deformations, limited control feedback, reduced control bandwidth, stability issues, underdamped modes, and lack of precision in tasks involving working against external loads. These usually result in modeling and/or control challenges for such designs. Here, we introduce two new modeling approaches for continuum rods and actuators, a general reduced-order model (ROM), and a discretized model with absolute states and Euler-Bernoulli beam segments (EBA). These models enable us to perform more accurate simulation of continuum manipulators, as well as modeling 2D and 3D continuum geometries, something which has been missing in similar recent research [10]. In addition, a new formulation is presented for a recently intro- duced discretized model by [10, 18] which is based on EulerBernoulli beam theory and relative states (EBR).

In the following sections, first, a brief review on models for continuum manipulators is presented. Then, we discuss rigidbody kinematics and how they can be unified with variable curvature and discretized continuum kinematics, with relative and absolute (independent) states. A new general yet efficient reduced order solution for the rod backbone is discussed based on truncated series. Continuum manipulator dynamics is discussed where a lumped-system representation of the Cosserat rod theory and a new discretization method based on absolute (independent) states is presented. Experimental results from a (STIFFness controllable Flexible and Learn-able manipulator for surgical OPerations) continuum appendage [5] are used to investigate the computational performance and simulation accuracy of the discussed continuum rod models. Finally, a discussion and conclusion are presented.

\section{Materials \& Methods}

\section{A. Continuum Structures Mechanics: A Short Review}

If taking a theoretical approach in constrast to pure learning [23], combined reduced-order solution and learning [21] approaches, and byond the distinction between $2 \mathrm{D}$ vs. 3D and static vs. dynamic models, two key stages can be identified which determine the modeling strategy of a soft robot [2, 6, 16] (Table II. 1) Modeling assumptions for 1-a) the system kinematics, 1-b) system conservation law (system mechanics), and 1-c) material constitutional law (material mechanics). This stage results in a system of differential equations for the mechanics of a continuum media. 2) The method to solve this resulting system, which can be based on 2-a) direct, or 2-b) indirect methods. Here, we focus on modeling methods for 1-dimensional (1D) continuum elements, (continuum rods), as the most studied continuum structure in soft robotic research. 
TABLE I: Different elements of a model for continuum rods.

\begin{tabular}{l}
\hline \hline 1- Modeling Assumptions \\
a- Kinematics \\
i- Continuous Geometry \\
A- Variable Curvature (VC- finite strain assumption) \\
B- Truncated Series Shape Assumption \\
C- Constant Curvature (CC) \\
ii- Discretized Geometry \\
A- Series Rigid-Body Kinematics \\
B- Screw Theory \\
C- Forward Discretization of VC \\
b- Mechanics (Conservation Law) \\
i- Cossert Rod Method \\
ii- Principle of Virtual Work (PVW) \\
iii- Beam Theory \\
iv- Lagrange Dynamics \\
c- Material Mechanics (Constitutional Law) \\
i- Linear Elasticity (Hooke's Law) \\
ii- Finite Strain Theory (Neo-Hookean, Money-Rivlin, Gent, ...) \\
iii- Visco-Hyperviscoelastic \\
\hline 2- Solution \\
a- Direct Methods \\
i- Analytical Integration \\
ii- Numerical Forward Integration Steps \\
b- Indirect methods \\
i- Optimization Based Methods \\
ii- Finite Element Methods (FEM) \\
iii- Reduced Order Method (Ritz Method) \\
iv- Combination of the above \\
\hline
\end{tabular}

Two methods are widely used to describe continuum rod kinematics; 1-a-i) continuous kinematics, and 1-a-ii) discretized Kinematics. 1-a-i-A) Variable Curvature (VC- finite strains assumption) [24], 1-a-i-B) truncated series shape functions (Reduced Order Models- ROM) [7, 17], and 1-a-i-C) Constant Curvature (CC- as a subset of general shape function approach), which is probably the most simple and widely used assumption for soft manipulator modeling [26] report instances of employing continuous kinematics.

Instances of using discretized representation of such system kinematics are as followes. 1-a-ii-A) Employing series rigidbody kinematics (SRL), by simplifying a continuum rod as a hyper-redundant mechanism with finite but large enough number of segments, based on transformation matrices for consecutive but distinct rotational and translational joints [4, 18]. Methods based on 1-a-ii-B) Screw Theory [10] and 1-a-ii-C) forward discretization of VC differential equations (EBR- based on differential relative states) [18], where a skewsymmetric matrix of local curvatures/torsion vector is used to describe the local relative rotations along the backbone.

1-b-i) The Cosserat Rod method [24, 2], 1-b-ii) Principle of Virtual Work (PVW) [15], 1-b-iii) Beam Theory [15], and 1-biv) Lagrange Dynamics [8, 17, 4] are used to derive the system governing equation (conversational law). The material constitutional law (material mechanics) is usually derived based on 1-c-i) linear elasticity theory (Hooke's law) [24, 8, 16], 1-cii) finite strain theory (considering large strain in hyperelastic materials, such as Neo-Hookean [24, 15, 16, 18], MooneyRivlin, Gent [18], etc), or 1-c-iii) by considering hyperviscoelastic properties [17, 9].

Any combination of the above choices results in a system of Ordinary (ODE) or Partial Differential Equations (PDE) to be solved numerically based on the system initial and boundary conditions. Using shape functions or discretized kinematics results in PDEs with decoupled spatial and temporal domains where direct solutions based on 2-a-i) analytical, if possible, or 2-a-ii) numerical forward integration steps in spatial and time domain can be used to solve the resulting initial value problems [8, 15, 17]. Such systems turn into a Boundary Value Problem (BVP), if static solutions are sought. Although, forward integration is valid in such cases if distributed loads, e.g. body weight, are neglected [18].

Alternatively, indirect solutions (2-b) can be sought. 2-b-i) Optimization-based methods, i.e. single shooting [15, 16, 17], multiple shooting, and concatenation methods, are suitable for BVPs resulting from static models with general loads, or for learning the coefficients of an approximate series solution [7] or gains in a neural network model [23]. 2-b-ii) Finite Element Methods (FEM) or similar segmentation methods [3, 1] are suitable if spatial (kinematics) discretization methods are used where, instead of a forward integration over the spatial domain, a system of nonlinear equations is formed with a large but sparse coefficient matrix. The system equilibrium point in static cases or at every time step of a dynamic simulation is found by calculating the pseudo-inverse of the coefficient matrix, while satisfying all the geometrical, dynamical and optimal control constraints [3]. While considering truncated series solutions as the system kinematics 2-b-iii) reduced order [22] or Ritz [17] method for solving a PDE problem, different choices of weighting functions can be used to improve the accuracy of the solution, e.g. in the case of Ritz-Galerkin methods [25, 17]. Finally, 2-b-iv) a combination of the above methods can be used, usually for solving PDEs resulting from complex geometries.

As an example of general practice in many commercially available FEM solvers, Tunay used a discrete Galerkin method, where weighted governing equations are used to construct the FEM solution for pneumatic actuators with general deformation [25]. Sadati et al. used forward integration on spatial domain for the PDEs resulting from employing reduced order solutions for continuum manipulator kinematics, and then combined that with single shooting optimization method to find the system static solution under excessive external tip loads [15, 17]. Bieze et al. combined FEM and optimization methods to solve the closed-loop control problem of continuum manipulators [1]. Gazzola et al. combined FEM with forward integration on time-domain in dynamic simulations [6]. Thieffry et al. constructed a reduced order model based on dominant deformation modes that are found from multiple FEM based simulations of a system under different loading conditions. The coefficients of such solution were then optimized to solve for general cases [22]. Duriez, Bieze and Thieffry used a SOFA FEM modeling package for real-time dynamic simulation of soft structures [3, 1, 22].

In a comparative study with experimental results with a single module STIFF-FLOP appendage [16], we have recently shown the advantage of a lumped system approach (1-a-ii-A \& 1-b-iv \& 2-a-i) for dynamic analysis and traditional control design, CC and modified CC (1-a-i-C \& 1-b-ii \& 2-a-i) for 
considering structural complexity and design parameter study, Cosserat rod theory (1-a-i-A \& i-b-i \& 2-b-i) for accuracy in general cases, and reduced order series solutions (1-a-i-B \& 2 -b-i) for real-time performance. The study in [16] is based on 1-c-i. We showed that combining reduced order kinematics, the Cosserat rod mechanics, numerical integration on spatial domain and optimization based solution (1-a-i-B \& 1-b-i \& 2a-ii \& 2-b-i) produces most of the aforementioned advantages, i.e. accuracy, simple control design, real-time performance, considering structural complexity for a single STIFF-FLOP appendage in planar motion with excessive external load at the tip [17]. Further, we generalize our solution for multisegment arms in general 3D dynamic motion and compare the accuracy and numerical performance of the results with models with other assumptions. Additionally, the discretization method presented by Renda et al. [10], which is based on Screw Theory and transformation matrices, is modified to use absolute (independent) states to achieve discretized models for multi-dimensional continuum geometries with a large number of states and significantly improved numerical efficiency.

Additionally, the Reduced Order Models (ROM) for continuum rod kinematics discussed by Godage et al. [7, 8] based on the pressure chambers' length for a pneumatic soft manipulator. The presented solution is hard to interpret, results in complicated dynamic derivations, and the mechanical coupling between the actuation chambers' input pressure and length are not considered. The large number of coefficients that should be learned through experimental trials is another drawback of such a method. In our previous work, we showed the advantageous numerical performance and accuracy of using a truncated Lagrange polynomial series passing through some arbitrary points along the backbone [17]. The proposed solution is easy to interpret for shape estimation and controller design, since the used polynomial is constructed using Cartesian coordinates of physical points, and has small number of states ( 6 for a short appendage consisting of Cartesian coordinates of 2 points at the appendage tip and mid-length). Both the discussed methods by Godage et al. [7, 8] and Sadati et al. [17] solve the singularity problem of using Constant Curvature and rotation matrix representations. However, we used the CC assumption to compensate for the imaginary torsion of a Frenet-Serret frame and to find the physical torsion of the appendage cross-section based on the input chambers' pressure. In addition, the cross-section shear was neglected and a mean axial strain is assumed along the backbone. The Beam theory was used for static modeling and the PVW for dynamic modeling, based on Ritz and RitzGalerkin solutions. Duriez, Bieze and Thieffry have recently generalized the same concept to modeling and control of complex continuum geometries by extracting the dominant deformation maps using the SOFA FEM package [3, 1, 22].

In this paper, we continue our efforts toward framing accurate and real-time modeling methods with minimal modeling and control states that are suitable for controller design. Two new modeling approaches are presented for continuum manipulators; I) a general reduced-order model (ROM) capable

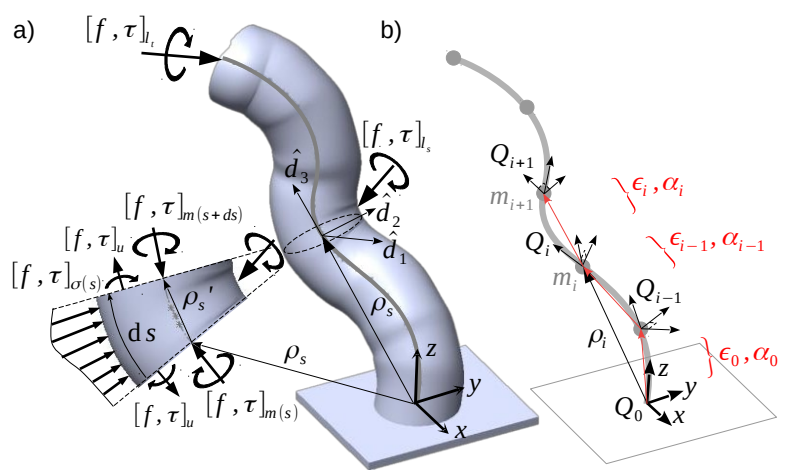

Fig. 1: a) Variable curvature kinematics and free body diagram of Cosserat rod method for one differential element along the continuum backbone. Subscript $\left({ }_{l}\right),\left({ }_{u}\right)$, and $\left(_{\sigma}\right)$ are for the external loads, loads due to internal pressures and/or tendons' tension, and distributed loads, e.g. due to gravity, respectively. b) Discretized VC kinematics and lumped mass representation of Cosserat rod method for the same continuum rod as in (a).

of capturing full motion of a continuum backbone, and II) a discretized model with absolute states and Euler-Bernoulli beam segments (EBA) to improve the numerical performance of simulating long continuum manipulators. The above models alongside SLR and EBR models, based on a new formulation are implemented in a recently developed Matlab software package, named TMTDyn, to simplify their implementation and comparison with real experimental results from a STIFFFLOP pneumatic continuum appendage.

In the following sections, first the continuum-body system kinematics using quaternions and the reduced-order method of using truncated polynomial series are described. Then, the TMT dynamics of such systems are discussed. Finally, we explain how to derive the lumped-system equivalence of Cosserat rod and reduced-order methods with relative and absolute (independent) states.

\section{B. VC Kinematics for Continuum Rods}

We use 1-dimensional (1D) continuum elements, i.e. continuum rods, as the basis of modeling continuum geometries in this work. Using the Cosserat rod method, which considers all six translational (strains- $\xi$ ) and rotational (curvatures/torsion$\zeta$ ) differential states, is beneficial for 1D continuum elements. VC kinematics and the Cosserat rod theory are used to model the static mechanics of continuum rods, based on rotation matrices [6] and quaternion [24, 2] representation of rotations. In the past, we used Variable Curvature (VC), based on rotation matrices, and Beam theory to investigate continuum manipulator mechanics in static and dynamic motions [16, 15, 17]. We showed that employing a beam theory approach simplifies solving the BVP for static analysis of a continuum rod with numerical optimization-based or reduced order model methods. However, they are not a good candidate as a part of a unified framework for modeling hybrid systems and especially in a lumped system approach framework.

Here, the equations are derived in a local physical curvilinear coordinates $\left[\hat{d}_{1}, \hat{d}_{2}, \hat{d}_{3}\right]$, where $s$ is a variable for axial po- 


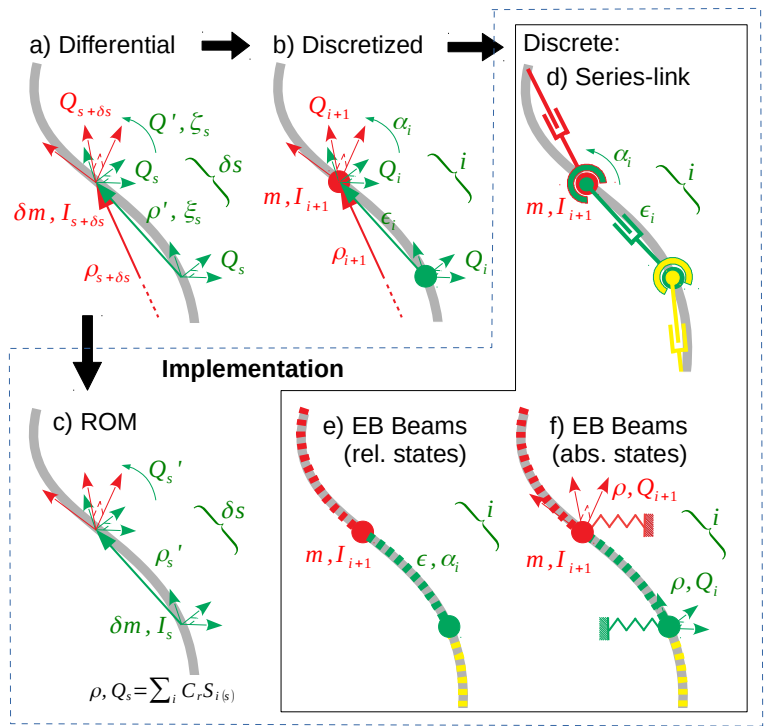

Fig. 2: Different modeling assumptions for a continuum rod. a) Rod differential Variable Kinematics (VC), b) discretized VC framework, c) Reduced Order Model (ROM) based on a polynomial series solution for the backbone kinematics, d) equivalent highly-articulated series rigid link mechanism, e) discretized model with relative states from Euler-Bernoulli (EB) beams, f) discretized model with absolute states (w.r.t. reference frame) and EB beam compliant connections. Backbone is shown by a continuous gray line and dashed curves are EB beam sections.

sition along the backbone curve, $\hat{d}_{3}$ is tangent to the backbone, and at the rod base we have $\left[\hat{d}_{1}, \hat{d}_{2}, \hat{d}_{3}\right](s=0)=[\hat{i}, \hat{j}, \hat{k}]$ (Fig. 1 a). The rod backbone spatial configuration in Cartesian coordinates $\left(\rho_{s}\right)$ and $1 \times 4$ rotation quaternion unit vector $\left(Q_{s}=\left[Q_{0}, Q_{\rho}\right]\right)$, expressed in global coordinates $([\hat{i}, \hat{j}, \hat{k}])$, are derived according to $\mathrm{VC}$ as in [11, 24]

$$
Q_{s}^{\prime}=Q_{s} \times\left[0, \zeta_{s}\right] / 2, \rho_{s}^{\prime}=Q_{s} *\left(\xi_{s}+[0,0,1]\right),
$$

where $\xi$ and zet $a$ are the curve local strain and bending/torsion vectors, superscript ( $\left.{ }^{\prime}\right)$ is used for spatial differentiation by $s$, $\times$ represents quaternion multiplication and $*$ is the operator for quaternion rotation of a vector as in [11]. The implementation of quaternion rotation $(*)$ is more computationally expensive than using rotation matrices $R_{s}$ as $R_{s}^{\prime}=R\left[\zeta_{s}\right]_{\times}$and $\rho_{s}^{\prime}=$ $R\left(\xi_{s}+[0,0,1]\right)$, where [ $]_{\times}$denotes the standard mapping from $\mathbb{R}^{3}$ to $\sim \rtimes(3)[2]$. However, quaternions are reported to be better in terms of numerical integration accuracy, and preserve frame orthogonality and vector length [24, 11].

\section{Discretized VC Kinematics}

First-order forward difference is probably the simplest way for discretization of a differential equation, where the differential terms are substituted with the finite difference of the system states subject to small increments of the free variables, here spatial states. Discretized versions of Eq. 1 with rotation matrices are discussed in [20, 10, 18]. Shiva et al. used first order discretization, $R_{i+1}=R_{i}\left(\zeta_{i} \Delta s+\mathbf{I}_{[3 \times 3]}\right)$ ( $i$ is the element numerator,), which is probably the simplest assumption, failing to conserve the principal properties of a rotation matrix. Renda et al. used the same method in the context of screw theory as $R_{i+1}=R_{i} e^{\zeta_{i} \Delta s}$, where $e^{\zeta \Delta s}=\zeta \Delta s+\mathbf{I}_{[3 \times 3]}$ [10]. Takano et al. used the most accurate representation for $\zeta$ with Euler angles (three consecutive rotations around local frame principle unit vectors) with 1-2-3 $(x-y-z)$ order $\left(R_{x y z_{\zeta}}=R_{x_{\zeta_{1}}} R_{y \zeta_{2}} R_{z_{\zeta_{3}}}\right.$ ), as $R_{i+1}=R_{i} R_{x y z_{\zeta_{i}}}$. A similar representation is discussed by Shiva et al. in [18], appendix section, arguing that the order of the rotations is not important as long as small enough elements are considered along with the backbone (infinitesimal curvatures/torsion). They showed that using any of the above methods does not affect the accuracy of modeling a short appendage with beam theory, even for large deformations.

Here we use the first representation used by Shiva et al. and Renda et al., since it is easy to interpret its inverse; i.e. $\zeta_{i}=R_{i}^{\top}\left(R_{i+1}-R_{i}\right) / \Delta s$, which is necessary for modeling a continuum rod with absolute (independent) modeling states. Using quaternions and their properties, the final form of the discretized equations are

$$
\begin{aligned}
Q_{i+1} & =Q_{i} \times\left[1, \alpha_{i} / 2\right], \\
\rho_{i+1} & =Q_{i} * \epsilon_{i}+\rho_{i},
\end{aligned}
$$

for VC kinematics and

$$
\begin{aligned}
{\left[0, \alpha_{i}\right] } & =2 Q_{i}^{-1} \times\left(Q_{i+1}-Q_{i}\right), \\
\epsilon_{i} & =Q_{i}^{-1} *\left(\rho_{i+1}-\rho_{i}\right),
\end{aligned}
$$

for their inverse. Following the definition of engineering strains, $\alpha=\zeta \Delta s$ is the local bending/twist angle vector, and $\epsilon=\Delta \rho=(\xi+[0,0,1]) \Delta s$ is the deformed local position/translation vector. Notice that $Q_{i}$ is the absolute orientation quaternion at each point, but $\alpha$ describes the relative orientation of consecutive elements. The above equations show that the deformation of a discretized element can be modeled as a 3D translational joint with state space $\epsilon$ and initial value $\epsilon_{0}$, followed by a 3D rotational joint with state $\alpha$, initial value $\alpha_{0}$ and quaternion representation of $Q_{\alpha}=[1, \alpha / 2]$ (Fig. 1 $\mathrm{b}$ ).

Having an element's initial bending/twist angle $\left(\alpha_{0}\right)$ and a local translation vector $\left(\epsilon_{0}\right)$, the local deformation of the discretized geometry $\left(\Delta \alpha=\alpha-\alpha_{0}\right.$ and $\left.\Delta \epsilon=\epsilon-\epsilon_{0}\right)$ can be calculated for deriving the element viscoelastic mechanical action due to system deformation.

\section{Reduced Order Kinematics}

As mentioned earlier, our reduced order method based on a truncated polynomial solution in [17] relays on estimation of the cross-section torsion based on CC assumptions and internal pressures/tensions of the manipulator actuators. Here, we drop using Frenet-Serret frames and present a new general ROM approach to account for the cross-section local strains, as well as dealing with curvatures/torsion without any secondary assumptions, e.g. CC. Additionally, a simple polynomial is used instead of a Lagrange polynomial which results in simpler and faster derivation of the system kinematics. An inverse linear problem is solved to find the initial value of the 
polynomial coefficients based on the position and orientation of some nodes along the rod backbone. The final solution is more suitable for Cosserat rod and PVW methods. We assume that the manipulator geometry is defined by 6 truncated polynomial series of order $n_{r}+1$ for position and $n_{r}$ for orientation map (3 for $\rho$ vector and 3 for vector part of local frame quaternion representation of orientation $Q_{\rho}$ ), as

$$
\left[\rho, Q_{\rho}\right]=\sum_{i=1}^{n_{r}}\left(C_{r} S_{i}\right)+S_{0},
$$

where $Q_{\rho}$ is the vector part of the quaternion representation of backbone orientation $\left(Q=\left[Q_{0}, Q_{\rho}\right]\right)$ with $Q_{0}=$ $\sqrt{1-Q_{\rho} Q_{\rho}^{\top}}, n_{r}$ is the polynomial order, $C_{r\left[6 \times n_{r}\right]}$ is the polynomial coefficient matrix which is considered as the system modeling states, $S_{i}=[s, s, s, 1,1,1] s^{i}$ and $S_{0}=$ $[0,0, s, 0,0,0]$ are the shape function matrices that satisfy the rod base boundary conditions, i.e. being perpendicular to the base. For cases where the nodes rotate more than $180[\mathrm{deg}]$ around any axis, the full quaternion $(Q)$ with all four elements is used instead of $Q_{\rho}$ to avoid any singularity. In this case, the quaternion conjugate should be handled as $Q^{-1}=\left[Q_{0},-Q_{\rho}\right] / \sqrt{Q Q^{\top}}$. Quaternion representation of rotations ans system kinematics are handled as in [11]. Defining $S \equiv S_{1: n_{r}}$, we can rewrite the above equation as $\left[\rho, Q_{\rho}\right]=C_{r} S+S_{0}$. The state (coefficient matrix) initial values $\left(C_{r 0}\right)$ are found based on position $\left(\rho_{0}\right)$ and orientation $\left(Q_{\rho 0}\right)$ of a few points along the manipulator backbone $\left(s_{r}\right)$ by solving the following inverse problem, $C_{r 0}=\left(\left[\rho_{0}, Q_{\rho 0}\right]-S_{0_{s_{0}}}\right) S_{s_{0}}^{-1}$. The above inverse problem can be solved efficiently using Matlab inv function. $\rho_{0}$ and $Q_{\rho 0}$ can be simply measured from experimental observations using magnetic or visual trackers. For the local strain $\xi$ and curvatures/torsion $\zeta$, from Eq. 1 and similar to the inverse map in Eq. 2, we obtain

$$
[0, \zeta]=2 Q^{-1} \times Q^{\prime}, \xi=Q^{-1} * \rho^{\prime}-[0,0,1] .
$$

$\xi$ and $\zeta$ are used to calculate the mechanical action of the rod structural compliance.

The next step is dealing with the continuum manipulator dynamics while incorporating the discussed kinematics. To this end, in the next section, we adopted a differential form for the TMT method of deriving Lagrange EOM.

\section{E. Continuum Body Dynamics}

EOM for a rigid system can be derived using TMT representation of Lagrange method as [14]

$$
\sum_{i=1} \bar{M}_{i} \ddot{q}=\sum_{i=1} \bar{d}_{i}-\sum_{i} w_{i}^{\top}
$$

where, $q$ is the system states, $\bar{M}=T^{\top} M T$ is the system inertial matrix $(M)$ in state scape $(q), \bar{d}=T^{\top}\left(-M(T \dot{q})_{, q} \dot{q}+f_{g}\right)$ is the other inertial and gravity $\left(f_{g}\right)$ terms in EOM, ${ }_{, q}$ is partial differentiation w.r.t. $q, T=\chi_{q}$ is the transformation Jacobian between Cartesian $(\chi)$ and state space $(q)$ coordinates, $w=$ $T_{f}^{\top} f$ represents other mechanical actions in the system due to viscoelastic elements, external, internal, and body forces, etc., $f$ is the action exerting force, $T_{f}$ is the Jacobian transformation of the action exerting point from Cartesian to state space, $X_{, x}=\partial X / \partial x$, and the summation is over the total number of inertial elements and other actions in the system. Eq. 6 can be easily adapted for a discretized continuum rod using the lumped mass method where the differential form of TMT terms (replacing $\sum X$ with $\int \mathrm{d} X$ ) can be used [17]. For each ROM element in the system, the above spatial integrals can be handled with a numerical forward integration method, e.g. trapezoidal rule implemented in Matlab software trapz function, in each integrating time step of differential form of Eq. 6. To this end, we start with the differential form and then differential lumped mass representation for Cosserat rod model.

\section{F. Discretized Continuum Dynamics with Relative States}

Discretizing Cosserat rod mechanics [24], a highly articulated system with length $l, n_{d}$ elements, and relative states $(q=[\epsilon, \alpha])$ is formed with the kinematic relation expressed in Eq. 2. $M$ and $T_{m}$ are found by substituting $\rho_{i}$ and $Q_{i}$ from Eq. 2 to find the TMT inertial terms as in Eq. 66 The external loads are handled based on their exerting point, found from Eq. 2 In such systems, beam elasticity and damping, and the internal pressure/tendon tension acts parallel to the states $q$, so we set the states elastic $k$ and viscous damping $\mu$ coefficients as $k \mid \mu_{q}=\left[k\left|\mu_{\epsilon}, k\right| \mu_{\alpha}\right]$ and $f_{l_{q}}=\left[f_{u}, \tau_{u}\right]$, and follow the relevant procedure for compliance elements and loads explained above. Finally, the above terms are used alongside other terms in Eq. 6. The proposed procedure is easy to implement; however, the derived equations tend to be complex for more than ten elements, which results in long segments, resulting in inaccurate results, slow derivation and simulation [13, 20]. The method is not suitable for large system models.

\section{G. Discretized Continuum Dynamics with Absolute States}

To avoid complex derivations for a high number of elements, we may assume the discretized system states to be the lumpedmasses' Cartesian positions and take the vector part of their unit quaternion orientation as $q=\left[\rho, Q_{\rho}\right]$. $Q_{0}$ is derived based on $Q_{\rho}$ to form a unit quaternion. The system kinematics is the same as $q$ and increasing the number of elements does not increase the complexity of the derivations. $M$ and $T_{m}$ are found based on $\rho$ and $Q$, to find the TMT inertial terms of Eq. 6 differential form. The external loads become loads directly acting on system states $f_{l_{q}}=f_{l}$. The inverse map presented in Eq. 3 is used to derive $\epsilon_{i}$ and $\alpha_{i}$, based on which the beam elasticity $\left(w_{k}\right)$ and damping $\left(w_{v}\right)$, and the internal pressure/tendon tension $\left(w_{u}\right)$ actions are calculated as

$$
\begin{aligned}
\chi_{b} & =\left[\epsilon_{i}, \alpha_{i}\right], T_{b}=\chi_{b, q}, \\
w_{k}^{\top} & =T_{b}^{\top} k_{\epsilon \mid \alpha}\left(\chi_{b}-\chi_{b_{0}}\right), \\
w_{v}^{\top} & =T_{b}^{\top} \mu_{\epsilon \mid \alpha}\left(T_{b} \dot{q}\right)^{\nu}, w_{u}^{\top}=T_{b}^{\top}\left[f_{u}, \tau_{u}\right],
\end{aligned}
$$

where $\chi_{b_{0}}$ is the beam initial position vector and bending/twist angle that can be fund based on the system states' initial 

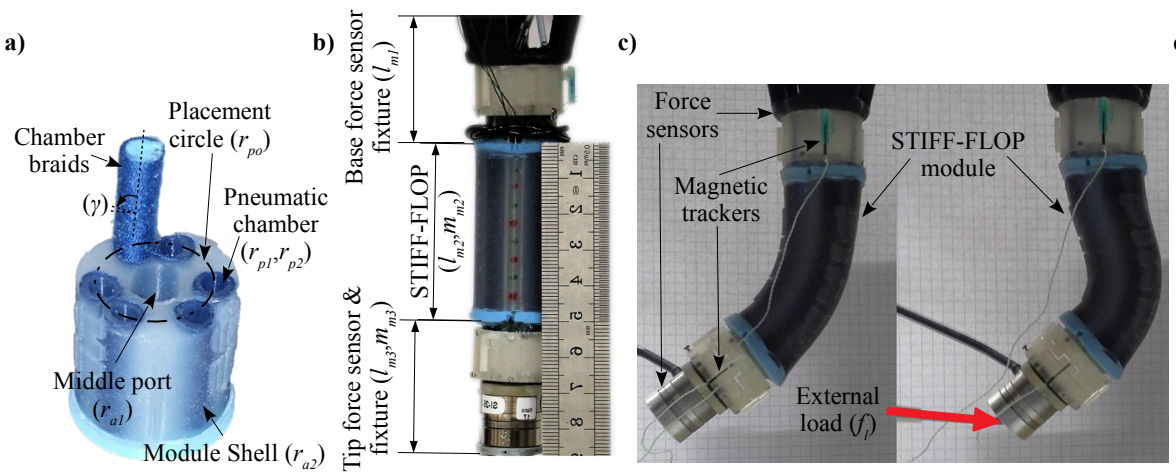

\begin{tabular}{l|r} 
Sym. & Value \\
\hline$r_{a_{1}}[\mathrm{~mm}]$ & 4.5 \\
$r_{a_{2}}[\mathrm{~mm}]$ & 12.5 \\
$r_{p}[\mathrm{~mm}]$ & 2.5 \\
$r_{p_{o}}[\mathrm{~mm}]$ & 8.5 \\
$l_{m_{2}}[\mathrm{~mm}]$ & 50 \\
$l_{m_{3}}[\mathrm{~mm}]$ & 14.5 \\
$m_{2}[\mathrm{gr}]$ & 28 \\
$m_{3}[\mathrm{gr}]$ & 13.47 \\
$\gamma[\mathrm{deg}]$ & 87 \\
$E[\mathrm{KPa}]$ & 205 \\
$\sigma\left[\mathrm{Kg} / \mathrm{m}^{3}\right]$ & 1820 \\
$\mu_{\epsilon}[\mathrm{Ns} / \mathrm{m}]$ & $0.1-100$ \\
$\mu_{\alpha}[\mathrm{Nms} / \mathrm{rad}]$ & $0.5-1 \mathrm{e}-5$ \\
$\nu$ & 2
\end{tabular}

Fig. 3: a) Structural design, b) parameters and c) sequences of experiments with a pneumatically actuated STIFF-FLOP continuum appendage. d) The structural parameters and their units are based on experimental measurments.

condition $q_{0}$ as $\chi_{b_{0}}=\chi_{b}\left(q_{0}\right)$. The above terms are used alongside other terms in Eq. 6. The proposed method allows handling a large number of elements. To the best of our knowledge, this is the first time that such a discretization method is used for modeling an actuated continuum manipulator, as well as its integration to a unified hybrid system modeling framework. However, similar discretization methods are widely being used to solve hyperbolic PDEs numerically. Meeting Courant-Friedrichs-Lewy condition is necessary for converging the solution which usually results in systems with very large number of elements and hence slow performance [19]. We do not analyze the convergence criteria in this paper, but a comparison with experimental results and the other presented methods in this paper are provided later.

\section{H. Reduced Order Model Dynamics}

In the case of reduced order model kinematics, the system spatial and temporal domains are decoupled. So we keep the differential form of Eq. 6, and perform a forward numerical integration over the ROM terms in each time step of the final system EOM numerical temporal integration. Here the states are the elements of $C_{r}$ in Eq. 4 which gives $6 \times n_{r}$ states. The system kinematics is presented in Eq. 4 as $\chi_{s}=\left[\rho_{s}, Q_{\rho_{s}}\right]$, and $T_{m}$ is found based on $\rho_{s}$ and $Q_{\rho_{s}}$. for $\mathrm{d} M$ we have, $\mathrm{d} m=\sigma a \mathrm{~d} s$ and $J_{m_{s}}$ is found based on the second moment of inertia for planar objects with the shape of the rod cross section. TMT differential terms are found as in [17]. The contact point kinematics of an external load at location $s_{l}$ along the backbone is found by substituting $s=s_{l}$ in Eq. 4 . Using the inverse map in Eq. 5 to find $\xi, \zeta$, the differential form of Eq. 7 is used to find the action derivatives for viscoelastic structure and internal pressures/tendon tensions. This method does not suffer from discretization inaccuracy; however, the modeling accuracy depends on the order of the polynomial, while a higher number of terms does not necessarily improve the accuracy. Initial bent configurations, rods with initial arbitrary geometries are easy to handle, by choosing appropriate values for $\chi_{s_{0}}$.

The discussed modeling frameworks for soft robots have enabled us to incorporate their states into the traditional modeling and control frameworks of rigid body dynamics. In the next section, experimental results from random pressurization of a pneumatic continuum appendage (STIFF-FLOP) are compared with the simulation results from ROM, SRL, EBR, and EBA modeling approaches. We have implemented these methods in our recently developed software package, named TMTDyn [14, 13]. The methods complexity and numerical performance are compared based on the derivation and optimization time of forming the system EOM in a symbolic form, and numerical simulation times of the experimental scenarios.

\section{NumericAl Simulations \& EXPERIMENTS}

\section{A. Experimental Setup \& Procedure}

Results for dynamic motion of a single STIFF-FLOP continuum appendage in presence of external loading are used to verify and compare the presented modeling methods in comparison to experiments. A STIFF-FLOP (STIFFness controllable Flexible and Learn-able manipulator for surgical OPerations) module [5] is a pneumatic continuum appendage (Fig. 3). The same setup as in [18] is used to conduct the experiments with and without external load at the appendage tip. Sample sequences of the experiments with and without tip external loads are shown in Fig. 3 c . The measured and identified structural parameters of the experimental setup are presented in Fig. 3. d. The explanation for how to consider the actuation chamber arrangement in calculating the crosssection second moment of area, manipulator mass and inertia, input pressure action, and hyper-elasticity of the manipulator are provided in [17, 13].

\section{B. Experimental Results \& Discussion}

Two sets of experiments were carried out with and without external load at the manipulator tip, and for different input pressures. Each experiment takes about 55 [s] and dynamic data for the actuator inputs, the manipulator tip position, orientation, and force were recorded. Sample recordings from the two experiments in comparison to simulation results from EBR model (Euler-Bernoulli beam model with Relative states) with $n_{d}=4$ are presented in Fig. 4 Matlab ode $15 \mathrm{~s}$ was used to speed up the numerical integration in our simulation in this section. We observed that except for ROM (Reduced Order Model), other models accuracy are very sensitive to the change of cross-section parameters due to the hyper-elasticity assumption, in experiments with external tip load. Hence, we 

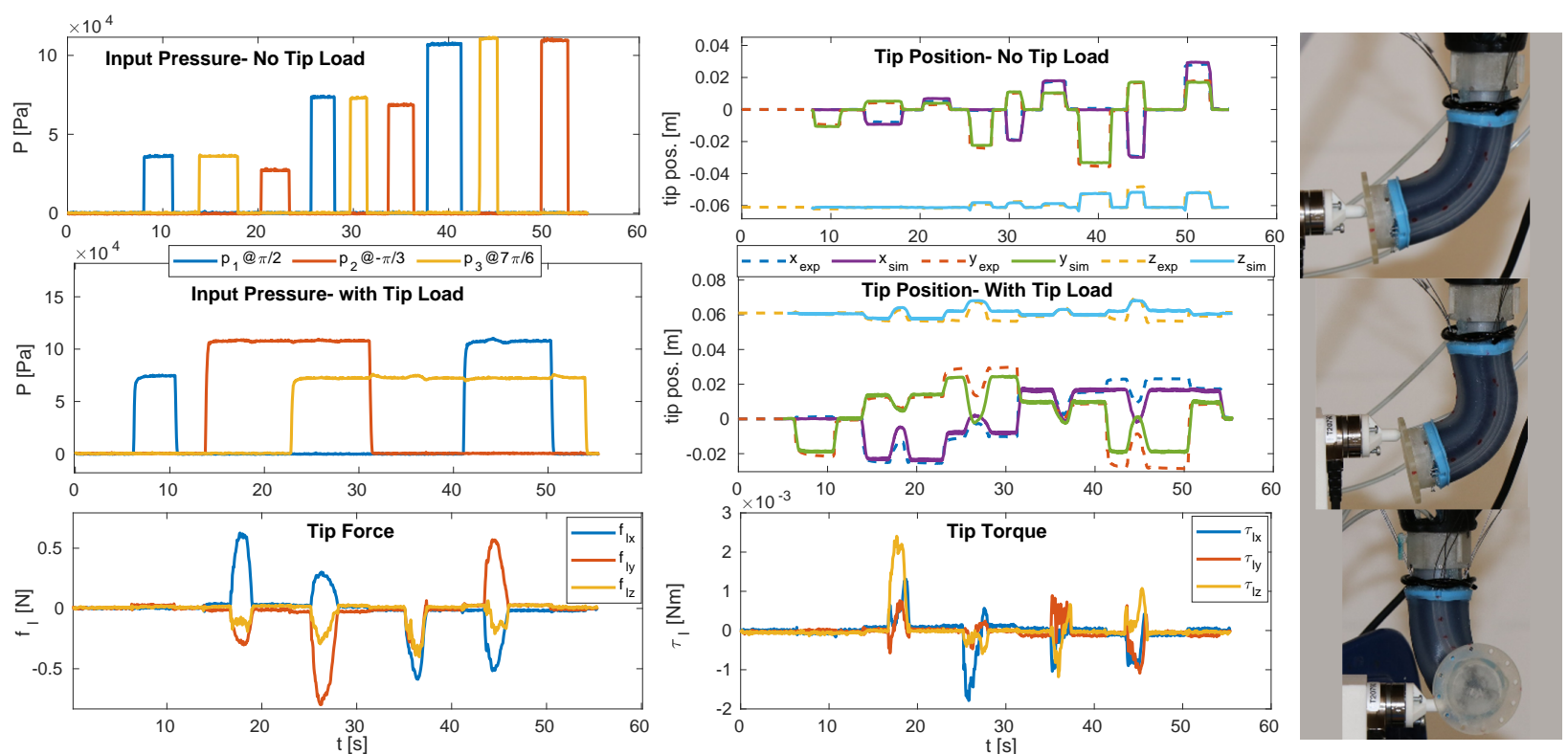

Fig. 4: Sample recordings from the two experiments with a STIFF-FLOP appendage in comparison to simulation results with EBR model with $n_{d}=4$. top) experiments with no external load, middle \& bottom) experiments with tip external loads. right) Sample sequences of experiments with external load.

neglected this change for the SRL (Series Rigid Link method), EBR, and EBA (Euler-Bernoulli model with Absolute states) cases in the externally loaded cases to avoid any numerical divergence in the simulations. Considering hyper-elasticity effects, if possible, improves the simulation results accuracy by up to \%6. This is in accordance with our previous observations in [17]. We set $\mu_{\xi}=0.1, \mu_{\zeta}=1 e-5$ for ROM and SRL, $\mu_{\xi}=100, \mu_{\zeta}=0.5$ for EBR, and $\mu_{\xi}=100, \mu_{\zeta}=0.01$ for EBA model. EBR model required higher damping coefficients to compensate the system sensitivity to rapid changes in the states in dynamic simulations. Considering these, EBR is more prone to be sensitive to numerical instability.

Fig. 5, 7 presents a comparison between computational performance and accuracy of these models with experimental results. ROM consumes the least memory and computer CPU time to derive the EOM, but EBA is the best in terms of CPU time for equation optimization. SRL is the worst in this regard. It takes hours to optimize the EOM for a system with more than three consecutive links. The change in system links, either to improve accuracy of a single rod model as presented here or in a system with multiple links, affects the EBA model CPU usage the least. For planar geometries (results are not provided here), a system with three times $n_{r}$ or $n_{d}$ of those presented here consumes the same memory and CPU time (Fig. 5p. As a result, EBA is the best model for systems with a large number of bodies. EBR and EBA have the best simulation time, as well as static and dynamic, performance. However, EBR showed to be very sensitive to sudden changes in the input pressure and external force values. High viscous damping values were considered to prevent exponentially growing errors (numerical analysis diverge) in this case. As a result, EBR simulation outputs were not reliable for fast dynamic motions. All the models show almost real-time performance (CPU time $<1$ [s]) except ROM which has the highest CPU time demand in simulations (Fig. 6. ROM presents relatively lower errors for a system with fewer number of states, even $n_{r}=1$, compared to other models, with $6[\mathrm{~mm}]$ absolute error (Abs. Err.) \& 9\% normalized error (Norm. Err.) for static motion and 3.5 [mm] \& $5.5 \%$ for dynamic motion in experiments without external loads (Fig. 6). These values are $12.5[\mathrm{~mm}] \& 19 \%$ for static and $9[\mathrm{~mm}] \& 14 \%$ for dynamic motion in presence of external tip loads (Fig. 7). The accuracy of other methods increases rapidly with higher number of states and even slightly surpass the accuracy of the ROM for experimental cases without external tip load (Fig. 6). The EBA model with 5 segments had 5.2 [mm] absolute error (Abs. Err.) \& 8.1\% normalized error (Norm. Err.) for static motion and $3[\mathrm{~mm}] \& 4.7 \%$ for dynamic motion in experiments without external loads (Fig. 6). This accuracy increase is less noticeable and even reverses in some cases (static motion with external load, Fig. 7) for the ROM. The ROM remains the most accurate model for experimental cases with external load. The EBA and EBR methods show similar performance for static analysis of the experimental case without external load while the EBA performs better for static analysis of cases with external load. However, the EBA method accuracy is much higher than the EBR method in dynamic simulations. The SRL method shows closer results to EBA, but we did not report the results for a model with more than three segments since it takes hours to derive and optimize their EOM. Results for EBR were not reliable for dynamic simulations since high viscous damping values filters parts of the dynamic motion. Our dynamic simulation results are more accurate than the static ones. However, we did not try to optimize the manipulator parameters or numerical analysis properties to find the best results, since this is not our main purpose in this study. It is possible to improve 

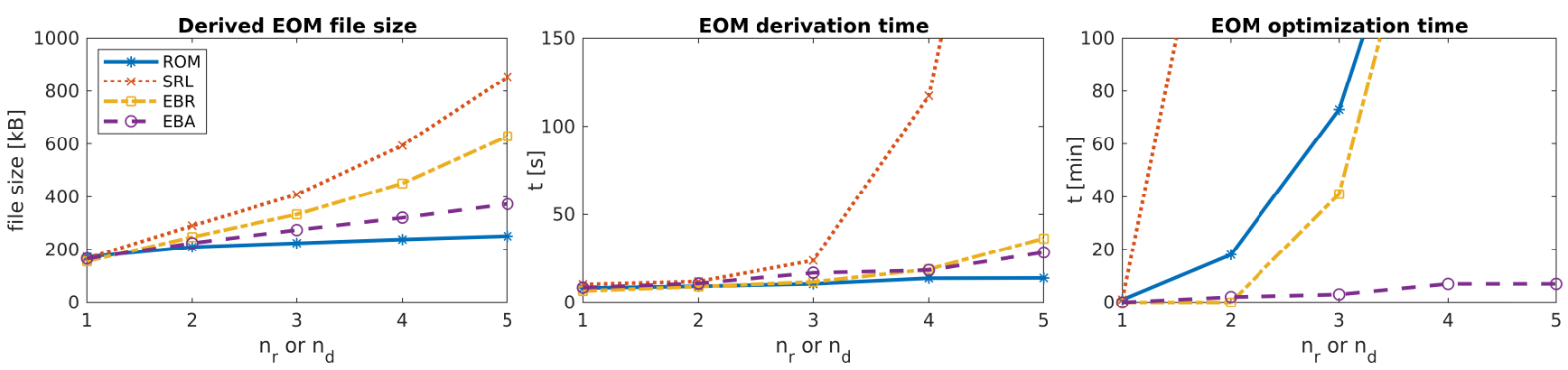

Fig. 5: Comparison between derivation complexity of different modeling assumption for a continuum rod based on (left) symbolic derivation file size, and (middle) derivation and (right) optimization time of derived EOM.
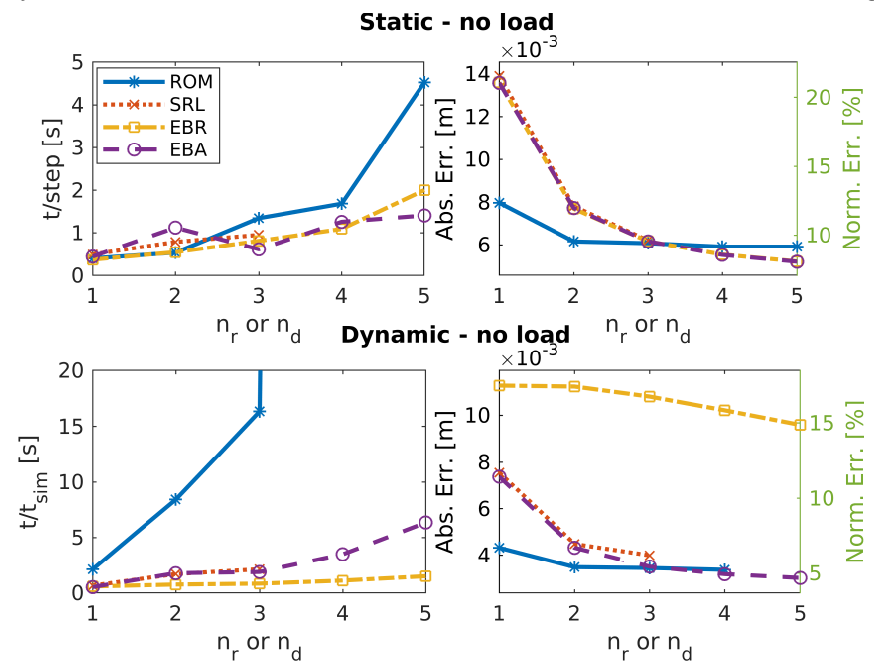

Fig. 6: Comparison between computational performance of simulations with different modeling assumption in comparison to experiments with a STIFF-FLOP continuum appendage with tip external load. (top) Static, (down) dynamic motion.

the accuracy by tuning these parameters or finding a way to effectively implement the hyper-elasticity assumption for all the modeling cases. Overall, we observed better accuracy of ROM, especially for a system with small number of states and cases with external tip loads, but better numerical performance of EBA, especially for large systems.

\section{CONCLUSION AND DISCUSSION}

In this paper, we develop two new models for continuum rods and actuators: a general reduced-order model (ROM), and a discretized model with absolute states and Euler-Bernoulli beam segments (EBA). These models enable us to perform more accurate simulation of continuum manipulators [10]. Furthermore, a new formulation is presented for a recently introduced discretized model by [10, [18] which is based on Euler-Bernoulli beam theory and relative states (EBR). The package is used to compare and validate the aforementioned modeling methods in comparison to experimental results on general motion of a STIFF-FLOP continuum appendage under external loads. We observed higher simulation accuracy (with as little as $8-14 \%$ normalized error) and numerical robustness (enabling consideration of material hyper-elasticity) of the
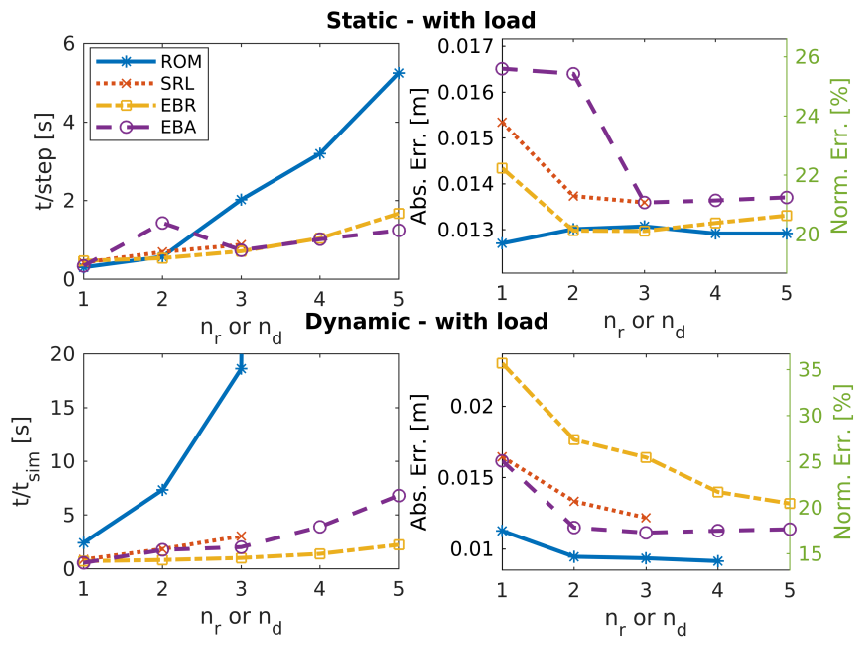

Fig. 7: Comparison between simulation accuracy of different modeling assumption for a continuum rod based on absolute and normalized \% error analysis in comparison to experiments with a STIFF-FLOP continuum appendage without any tip external load. (top) Static, (down) dynamic motion.

ROM model, while EBA is less computationally cumbersome to derive and simulate with near real-time performance. EBR shows high sensitivity to sudden changes in the system actuation inputs and external loads and relatively higher computational cost both in derivation and simulation. The lumped system approach for modeling continuum rods as a hyperredundant series-rigid-link system (SRL) is investigated as well. SRL has the highest computational cost to derive and optimize the system EOM. We plan to test our methods for hybrid force-position estimation and control of continuum manipulators in medical, inspection, and space robotics application.

\section{ACKNOWLEDGMENTS}

This work is supported by the U.K. Engineering and Physical Sciences Research Council (EPSRC) Grant EP/N03211X/2, European Union H2020 project FourByThree code 637095, Leverhulme Trust Project RPG-2016-345, the Royal Academy of Engineering research fellowship RF1516/15/11, and ERC Starting Grant No. 714562, granted to C. Bergeles. 


\section{REFERENCES}

[1] Thor Morales Bieze, Frederick Largilliere, Alexandre Kruszewski, Zhongkai Zhang, Rochdi Merzouki, and Christian Duriez. Finite Element Method-Based Kinematics and Closed-Loop Control of Soft, Continuum Manipulators. Soft Robotics, April 2018. ISSN 21695172. doi: 10.1089/soro.2017.0079. URL https://www. liebertpub.com/doi/abs/10.1089/soro.2017.0079

[2] Jessica Burgner-Kahrs, D. Caleb Rucker, and Howie Choset. Continuum Robots for Medical Applications: A Survey. IEEE Transactions on Robotics, 31(6):12611280, December 2015. doi: 10.1109/TRO.2015.2489500. URL http://ieeexplore.ieee.org/document/7314984/.

[3] Matteo Cianchetti and Arianna Menciassi. Soft Robots in Surgery. In Soft Robotics: Trends, Applications and Challenges, volume 9 of Biosystems \& Biorobotics, pages 75-85. Springer International Publishing, 1 edition, 2017. ISBN 978-3-319-46460-2. doi: 10.1007/ 978-3-319-46460-2. URL http://link.springer.com/10. 1007/978-3-319-46460-2_10

[4] Cosimo Della Santina, Robert K Katzschmann, Antonio Bicchi, and Daniela Rus. Dynamic Control of Soft Robots Interacting with the Environment. page 9, Livorno, Italy, 2018. IEEE.

[5] J. Fras, J. Czarnowski, M. Maciaś, J. Główka, M. Cianchetti, and A. Menciassi. New STIFF-FLOP module construction idea for improved actuation and sensing. In 2015 IEEE International Conference on Robotics and Automation (ICRA), pages 2901-2906, May 2015. doi: 10.1109/ICRA.2015.7139595.

[6] M. Gazzola, L. H. Dudte, A. G. McCormick, and L. Mahadevan. Forward and inverse problems in the mechanics of soft filaments. Royal Society Open Science, 5(6): 171628, June 2018. ISSN 2054-5703. doi: 10.1098/ rsos.171628. URL http://rsos.royalsocietypublishing.org/ content/5/6/171628.

[7] I. S. Godage, D. T. Branson, E. Guglielmino, G. A. Medrano-Cerda, and D. G. Caldwell. Shape functionbased kinematics and dynamics for variable length continuum robotic arms. In 2011 IEEE International Conference on Robotics and Automation (ICRA), pages 452457, Shanghai, China, May 2011. IEEE. doi: 10.1109/ ICRA.2011.5979607.

[8] Isuru S Godage, Gustavo A Medrano-Cerda, David T Branson, Emanuele Guglielmino, and Darwin G Caldwell. Dynamics for variable length multisection continuum arms. The International Journal of Robotics Research, 35(6):695-722, 2016.

[9] S. M. Mustaza, Y. Elsayed, C. Lekakou, C. M. Saaj, and J. Fras. Dynamic Modeling of Fiber-Reinforced Soft Manipulator: A Visco-hyperelastic Material-based Continuum Mechanics Approach. Soft Robotics, 2018.

[10] F. Renda, F. Boyer, J. Dias, and L. Seneviratne. Discrete Cosserat Approach for Multisection Soft Manipulator Dynamics. IEEE Transactions on Robotics, pages 1-
16, 2018. ISSN 1552-3098. doi: 10.1109/TRO.2018. 2868815.

[11] C. Rucker. Integrating Rotations Using Nonunit Quaternions. IEEE Robotics and Automation Letters, 3(4): 2979-2986, October 2018. ISSN 2377-3766. doi: 10.1109/LRA.2018.2849557.

[12] Daniela Rus and Michael T. Tolley. Design, fabrication and control of soft robots. Nature, 521(7553):467-475, 2015. ISSN 0028-0836. doi: 10.1038/nature14543. URL http://dx.doi.org/10.1038/nature14543.

[13] S M H Sadati, S E Naghibi, A Shiva, S Zschaler, H Hauser, I Walker, K Althoefer, and T Nanayakkara. AutoTMTDyn: A Matlab Software Package to Drive TMT Lagrange Dynamics of Series Rigid- and Continuum-link Mechanisms. 2018.

[14] S.M.Hadi Sadati, SE Naghibi, and M Naraghi. An Automatic Algorithm to Derive Linear Vector Form of Lagrangian Equation of Motion with Collision and Constraint. Procedia Computer Science, 76:217-222, 2015.

[15] S.M.Hadi Sadati, S. Elnaz Naghibi, Ali Shiva, Yohan Noh, Aditya Gupta, Ian D. Walker, Kaspar Althoefer, and Thrishantha Nanayakkara. A Geometry Deformation Model for Braided Continuum Manipulators. Front. Robot. AI, 4, 2017. ISSN 2296-9144. doi: 10.3389/frobt. 2017.00022. URL https://www.frontiersin.org/articles/ 10.3389/frobt.2017.00022/full

[16] S.M.Hadi Sadati, Seyedeh Elnaz Naghibi, Ali Shiva, Ian D. Walker, Kaspar Althoefer, and Thrishantha Nanayakkara. Mechanics of Continuum Manipulators, a Comparative Study of Five Methods with Experiments. In Towards Autonomous Robotic Systems, volume 10454, pages 686-702, Surrey, UK, 2017. Springer International Publishing. ISBN 978-3-319-64106-5 978-3-319-641072. doi: 10.1007/978-3-319-64107-2_56. URL http: //link.springer.com/10.1007/978-3-319-64107-2_56.

[17] S.M.Hadi Sadati, S. Elnaz Naghibi, Ian D. Walker, Kaspar Althoefer, and Thrishantha Nanayakkara. Control Space Reduction and Real-Time Accurate Modeling of Continuum Manipulators Using Ritz and Ritz-Galerkin Methods. IEEE Robotics and Automation Letters, 3 (1):328-335, January 2018. ISSN 2377-3766, 2377 3774. doi: 10.1109/LRA.2017.2743100. URL http: //ieeexplore.ieee.org/document/8014482/

[18] Ali Shiva, S.M.Hadi Sadati, Yohan Noh, J. Fras, Ahmad Ataka, Helge A. Wurdemann, Helmut Hauser, Ian. D. Walker, Thrishantha Nanayakkara, and Kaspar Althoefer. Elasticity vs. Hyperelasticity Considerations in Quasi-Static Modelling of a Soft Finger-like Robotic Appendage for Real-time Position \& Force Estimation | Request PDF. Soft Robotic Journal, (accepted, under print), 2018. URL https:/www.researchgate.net/ publication/328583306_Elasticity_vs_Hyperelasticity_ Considerations_in_Quasi-Static_Modelling_of_a_ Soft_Finger-like_Robotic_Appendage_for_Real-time Position Force Estimation 
[19] Robert D. Skeel and Martin Berzins. A Method for the Spatial Discretization of Parabolic Equations in One Space Variable. SIAM Journal on Scientific and Statistical Computing, 11(1):1-32, January 1990. ISSN 0196-5204, 2168-3417. doi: 10.1137/0911001. URL http://epubs.siam.org/doi/10.1137/0911001.

[20] Ryo Takano, Hiromi Mochiyama, and Naoyuki Takesue. Real-time shape estimation of Kirchhoff elastic rod based on force/torque sensor. In 2017 IEEE International Conference on Robotics and Automation (ICRA), pages 2508-2515, Singapore, Singapore, May 2017. IEEE. ISBN 978-1-5090-4633-1. doi: 10.1109/ICRA. 2017.7989292. URL http://ieeexplore.ieee.org/document/ 7989292/

[21] Maxime Thieffry, Alexandre Kruszewski, Christian Duriez, and Thierry-marie Guerra. Control Design for Soft Robots based on Reduced Order Model. IEEE Robotics and Automation Letters, pages 1-1, 2018. ISSN 2377-3766, 2377-3774. doi: 10.1109/LRA.2018. 2876734. URL https://ieeexplore.ieee.org/document/ 8496833/.

[22] Maxime Thieffry, Alexandre Kruszewski, Thierry-Marie Guerra, and Christian Duriez. Reduced Order Control of Soft Robots with Guaranteed Stability. In European Control Conference ECC18, Limassol, Cyprus, June 2018. URL https://hal.archives-ouvertes.fr/hal-01747433.

[23] T. G. Thuruthel, E. Falotico, F. Renda, and C. Laschi. Model-Based Reinforcement Learning for Closed-Loop Dynamic Control of Soft Robotic Manipulators. IEEE Transactions on Robotics, pages 1-11, 2018. ISSN 15523098. doi: 10.1109/TRO.2018.2878318.

[24] D. Trivedi, A. Lotfi, and C. D. Rahn. Geometrically Exact Models for Soft Robotic Manipulators. IEEE Transactions on Robotics, 24(4):773-780, August 2008. ISSN 1552-3098. doi: 10.1109/TRO.2008.924923.

[25] Ilker Tunay. Spatial continuum models of rods undergoing large deformation and inflation. IEEE Transactions on Robotics, 29(2):297-307, 2013. ISSN 1552-3098. doi: 10.1109/TRO.2012.2232532. 00000.

[26] R. J. Webster and B. A. Jones. Design and Kinematic Modeling of Constant Curvature Continuum Robots: A Review. The International Journal of Robotics Research, 29(13):1661-1683, 2010. ISSN 0278-3649. doi: 10.1177/ 0278364910368147. URL http://ijr.sagepub.com/cgi/doi/ 10.1177/0278364910368147. 